Escuela de Kinesiología, Facultad de Medicina, Universidad de Chile. aKinesiólogo (a).

Esta investigación no contó con financiamiento externo.

Recibido el 12 de noviembre de 2014, aceptado el 26 de diciembre de 2015.

Correspondencia a: Paulina López-Montecinos Escuela de Kinesiología Universidad de Chile Av. Independencia 1027, Independencia, Santiago. paulopezm@gmail.com

\section{Costo efectividad de un programa de rehabilitación cardiovascular modelo para personas post infarto agudo al miocardio en el Servicio de Salud Metropolitano Norte}

\author{
PAULINA LÓPEZ-MONTECINOS ${ }^{\mathrm{a}}$, JAME REBOLLEDO S. ${ }^{\mathrm{a}}$, \\ JOSÉ MIGUEL GÓMEZ L. ${ }^{a}$
}

\section{Cost effectiveness of a theoretical cardiac rehabilitation program after myocardial infarction}

Background: Secondary prevention programs are an essential part of comprehensive care of patients with cardiovascular disease (CVD), and its effectiveness in reducing morbidity or mortality has been proved. Aim: To determine the cost-effectiveness of a theoretical comprehensive cardiac rehabilitation (CCR) outpatient program after Myocardial Infarction, to be implemented in a Chilean Public Health System. Material and Methods: We designed a theoretical protocol of a CCR program based on recommendations of international guidelines, but adapted to local needs. A cost analysis was developed. Life years due to premature death were estimated with and without participation in CCR. The gained life-years and cost-effectiveness of the program were thus calculated. Results: The annual cost of cardiac rehabilitation center is $\$ 64,407,065$ Chilean pesos (CLP). The Incremental Cost Effectiveness Ratio (ICER) considering a reduction of late mortality of 25\%, is \$ 475,209.7 CLP per year of life gained. Since this figure is lower than one unit of per capita gross domestic product, the intervention is considered very cost-effective. Conclusions: A comprehensive cardiac rehabilitation program after myocardial infarction is very cost-effective in the context of its implementation in a public health service.

(Rev Med Chile 2016; 144: 456-464).

Key words: Cardiovascular Diseases; Cost Effectiveness; Health Care Costs; Myocardial Infarction; Rehabilitation.

\section{A}

ctualmente, el perfil epidemiológico de la población destaca una prevalencia mayor de enfermedades crónicas no transmisibles. En 2011 la mortalidad por enfermedad isquémica del corazón alcanzó una tasa de 41,7 por 100.000 habitantes a nivel nacional, con un total de 2.654 defunciones por infarto agudo al miocardio (IAM) en la Región Metropolitana y 7.197 defunciones a nivel nacional ${ }^{1}$. Además la alta recurrencia de
IAM (no fatal) determina una alta proporción de personas con alto riesgo cardiovascular, quienes sin un adecuado control significarán un elevado costo al sistema de salud ${ }^{2}$.

Los programas de rehabilitación cardiaca (PRC) secundaria de enfoque multidisciplinario han probado ser una estrategia efectiva en el cuidado integral de las personas con patología cardiovascular $^{3,4}$. El PRC provee una oportunidad alta- 
mente costo-efectiva de educar al paciente después de un episodio mórbido coronario y controlar los principales factores de riesgo cardiovascular ${ }^{4}$. Tanto los PRC basados sólo en ejercicio como las terapias integrales disminuyen significativamente la mortalidad general de personas con patologías cardiovasculares $^{5-11}$, ya sea con enfermedad coronaria como con insuficiencia cardiaca ${ }^{12}$. También la mortalidad por causa cardiovascular se reduce de manera significativa tras un $\mathrm{PRC}^{8}$.

En relación a la morbilidad cardiovascular post IAM, según estudios de la última década disminuye significativamente la angina inestable y cirugías de revascularización ${ }^{11}$, los factores de riesgo $\mathrm{CV}^{6-8}$ y los re-infartos ${ }^{9,10}$, existiendo variabilidad entre los estudios. Además existe un impacto positivo sobre la calidad de vida relacionada a la salud ${ }^{5-13}$.

Post IAM se recomienda una prescripción de ejercicio basada en una evaluación específica. La prescripción debe incluir frecuencia, intensidad, duración, modalidad y progresión ${ }^{3}$. El ejercicio constituye una importante herramienta terapéutica, pese a que el riesgo de complicaciones cardiovasculares aumenta transitoriamente durante el ejercicio vigoroso e infrecuente, en especial para personas que tienen enfermedad coronaria y son sedentarias ${ }^{13}$. Una prescripción inadecuada puede ser perjudicial para la población infartada. Es indispensable que el ejercicio sea guiado por un equipo con formación en rehabilitación cardiovascular y con preparación para asumir una emergencia ${ }^{4,15,16}$.

En Chile, el Grupo de Estudios Multicéntricos del Infarto (GEMI), recomienda las guías clínicas de la American Heart Association (AHA), European Society of Cardiology (ESC), además de la guía clínica del Ministerio de Salud Chileno (MINSAL) y de la Sociedad Chilena de Cardiología (SOCHICAR), para el manejo de pacientes con IAM. El GEMI recomienda implementar PRC secundarios para pacientes con riesgo moderado y alto, que incluyen ejercicio.

El año 2005 se incorpora a las Garantías Explícitas en Salud el diagnóstico, tratamiento y seguimiento del infarto agudo al miocardio. Se incorpora la garantía de acceso a prevención secundaria, lo que incluye profilaxis farmacológica; hipolipemiante, educación sobre hábitos de vida saludables, control adecuado de la hipertensión arterial y la diabetes. La garantía de seguimiento no explicita el acceso a un programa de ejercicio supervisado. En nuestro país, existen PRC basados en ejercicio físico realizados en su mayoría en instituciones privadas y concentrados en la Región Metropolitana ${ }^{18}$. Las razones de la falta de implementación de dichos programas podrían radicar en la falta de personal entrenado, limitaciones económicas, ausencia de espacio físico y en la baja percepción de beneficio clínico o rentabilidad ${ }^{19}$.

En Chile no se han publicado estudios de evaluación económica en este ámbito. Estudios internacionales señalan que la implementación de un PRC es costo-efectivo ${ }^{4,13,17,20}$.

El propósito de nuestro estudio es determinar la tasa costo-efectividad de un PRC modelo, que incluye ejercicio físico y consejerías, para contribuir al análisis económico de su implementación en el Sistema de Salud público chileno.

\section{Método}

Se realizó un estudio de costo-efectividad, considerando las recomendaciones de la Guía de Evaluación Económica del MINSAL (2013) ${ }^{21}$. Las alternativas a comparar son a) Trombolisis tras un IAM y b) Trombolisis más incorporación a un PRC.

Generamos un programa según recomendaciones de la AHA, Heart Foundation (Australia), ESCy National Institute for Health and Clinical Excellence (NICE). Se confeccionó un protocolo ambulatorio supervisado, técnicamente viable, ajustado a la realidad chilena y aprobado por un comité de expertos.

Para ajustar el programa a la población objetivo se estimó la demanda anual de personas sobrevivientes a un IAM en el Servicio de Salud Metropolitano Norte (SSMN).

Estimaciones y supuestos se presentan en la Figura 1. La población se caracteriza por ser mayores de 50 años, de predominio masculino, presentar uno o más factores de riesgo cardiovascular y enfermedades metabólicas. Según las bases de datos del DEIS (2011) 78\% de la población del SSMN son beneficiarios de FONASA.

La perspectiva del análisis de costo fue desde el servicio hospitalario, omitiendo gastos de pacientes. El horizonte temporal de los costos fue de un año, para dar cobertura a la demanda anual estimada, y en el caso de los efectos, se consideró la sobrevida a 24 meses tras el PRC, según un 


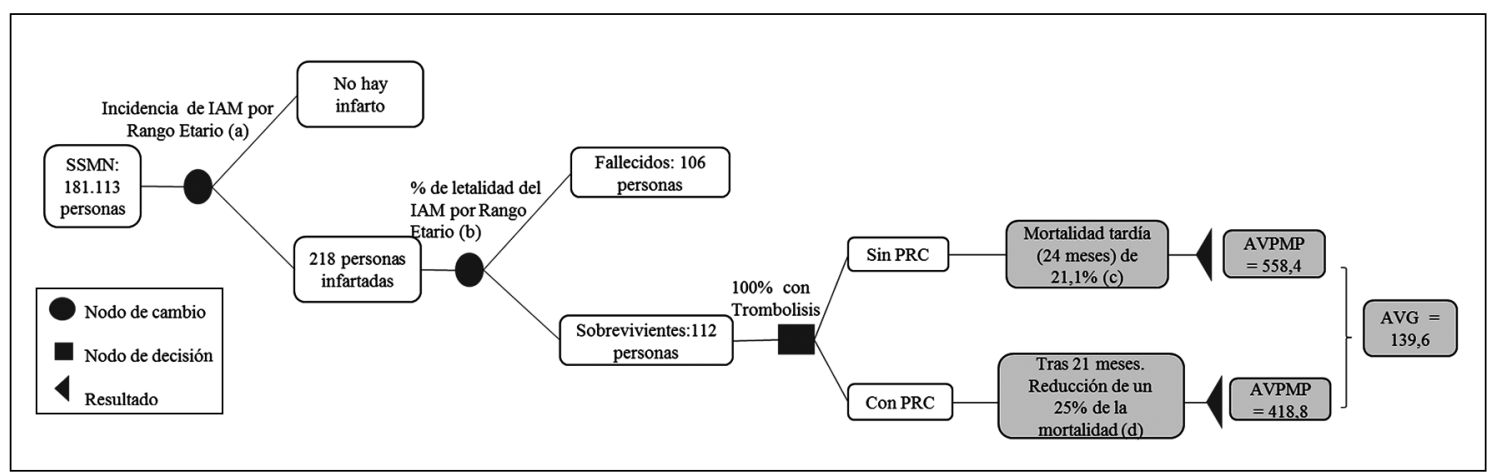

Figura 1. Árbol de decisiones: demanda anual de personas sobrevivientes a un IAM en el SSMN y estimación de años de vida perdidos por muerte prematura (AVPMP) y años de vida ganados (AVG) tras un PRC. (a) Unidad de Vigilancia de Enfermedades no Transmisibles. Departamento de Epidemiología. Reporte de Vigilancia de Enfermedades No Transmisibles (ENT) 2011. (b) Nazzal C, Alonso FT. Incidencia y letalidad por infarto agudo del miocardio en Chile: 2001-2007. Rev Med Chile 2011; 139: 1253-60. (c) Martínez SA, et al. Mortalidad post infarto del miocardio en Chile: Comparación de los registros de angioplastía primaria versus trombolisis. Rev Chil Cardiol 2010; 29: 29-36. (d) Lawler PR, et al. Efficacy of exercise-based cardiac rehabilitation post-myocardial infarction: a systematic review and meta-analysis of randomized controlled trials. Am Heart J 2011; 162: 571-84.

meta-análisis ${ }^{10}$, que incluye un análisis ajustable al contexto local en términos de calidad, población objetivo y características generales del PRC.

Se consideraron gastos directos e indirectos asociados a la prestación. Con respecto a los gastos directos, implican recurso humano y materiales audiovisuales. Los gastos indirectos se refieren a infraestructura, equipamiento, servicios básicos y gastos administrativos.

Los bienes de capital se valoraron de acuerdo a su valor de mercado, calculando el costo anual según los años de vida útil de los activos, obtenidos del Servicio de Impuestos Internos. El gasto en recursos humanos fue estimado acorde a la ley de remuneración 18.834 para profesionales de categoría B, considerando 5 años de experiencia y formación en la especialidad y la Ley 19.664 para profesionales de categoría $\mathrm{A}$, un médico cardiólogo con 9 años de experiencia.

Para estimar la eficacia en la reducción de la mortalidad se comparó el tratamiento convencional con trombolisis con el mismo tratamiento más el ingreso al PRC propuesto durante el primer año post IAM. Con base en la estadística nacional de mortalidad tardía (24 meses post-trombolisis), se calculó los años de vida perdidos por muerte prematura (AVPMP). Esta mortalidad tardía se verá reducida tras la intervención. Por lo tanto, se estimó los Años de Vida Ganados (AVG) por la aplicación de un PRC de 8 semanas de ejecución y 3 meses de seguimiento telefónico.

Se obtiene la Razón Costo Efectividad Incremental (RCEI o ICER en inglés) al relacionar el costo diferencial con los AVG, como indicador unidimensional de efectividad.

En relación al índice de costo-efectividad, se considera la clasificación empleada en el estudio de costo efectividad del MINSAL ${ }^{22}$. Si bien esta clasificación, se utiliza para estudios que expresan sus resultados en indicadores multidimensionales, como los AVAC (años de vida ajustados por calidad) y AVAD (años de vida ajustados por discapacidad), consideramos que el PIB per cápita es un umbral aceptable para AVG.

Finalmente se realizó un análisis de sensibilidad determinístico sobre la tasa de descuento, variables epidemiológicas y del potencial desempeño del programa. Se analizó la estimación de la demanda mediante el análisis bivariable de la incidencia y la letalidad del IAM. Los valores de variabilidad consideran las tendencias de incidencia y letalidad a 6 años ${ }^{23}$. Y un análisis univariable de los cambios de incidencia, letalidad, derivación y eficacia del PRC y su impacto sobre los resultados (AVG e ICER).

Por otro lado, se realizó un Análisis de Sensibilidad de tres escenarios, que considera: 1) menor derivación documentada (15\%) y adherencia de $50 \%$; 2 ) derivación y adherencia de $50 \%$ y 3 ) derivación $50 \%$ y adherencia $100 \%$. 


\section{Resultados}

\section{Programa modelo}

Según la incidencia y mortalidad de las comunas del SSMN, se obtuvo 112 personas sobrevivientes al IAM. Dado que las intervenciones son de carácter grupal, la capacidad máxima de atención es de 260 personas/año, superando la demanda. El PRC modelo se desarrolla en ocho semanas. Consiste en tres evaluaciones, tres sesiones semanales de ejercicio aeróbico y dos de ejercicio de sobrecarga, talleres grupales y consejerías individuales, además de un seguimiento telefónico por tres meses tras el alta. El programa combina la terapia de ejercicio (individualizado según la evaluación inicial) y estrategias conductuales a través de educación personalizada y grupal. La intervención se orienta al paciente y su cónyuge o familia. El detalle del PRC se observa en la Tabla 1.

\section{Análisis de costos}

El costo anual del centro de rehabilitación cardiaca es \$64.407.065 CLP (Tabla 2) considerando la capacidad máxima de atención. Como la demanda estimada fue de 112 personas, el costo por persona atendida corresponde a $\$ 575.063$ CLP.

\section{Estimación de AVG}

La Figura 1 muestra el esquema para la estimación de la efectividad. Considerando la mortalidad asociada al IAM declarada en el Reporte de Vigilancia de Enfermedades no Transmisibles (MINSAL, 2011) junto con la tasa de mortalidad tardía ( 2 años) tras un procedimiento de trombolisis $^{24}$, los AVPMP son 558,4 años (hombres 345,4 años y mujeres 213 años). Un PRC puede reducir en $25 \%$ esta mortalidad tardía, cambiando a 418,8 AVPMP para ambos sexos. Por lo tanto, los AVG (con una tasa de descuento de 0,03 ) serán 135,53 años, para ambos sexos (Tabla 3).

Dado que los resultados se evalúan a dos años plazo, se aplica una tasa de descuento de rango inferior $(0,0 \%)$ y superior $(6,0 \%)$ según recomendaciones de MINSAL ${ }^{21}$. Por lo tanto, los AVG considerando el ajuste temporal serían 139,6 y 34,5 respectivamente, lo que no afecta significativamente el resultado final de costo efectividad.

\section{Costo efectividad}

En consecuencia a que la alternativa B incluye la alternativa $\mathrm{A}$, el costeo realizado es incremental, es decir, sólo considera la diferencia atribuida a la implementación de un PRC. Del mismo modo los AVG son la diferencia obtenida entre los efectos

Tabla 1. Características generales del Programa de Rehabilitación Cardiaca modelo

\begin{tabular}{|c|c|}
\hline Aspecto & Característica \\
\hline Evaluaciones & $\begin{array}{l}\text { Se realizan } 3 \text { evaluaciones durante el programa; al ingreso, en la semana } 5 \text { y en la semana } \\
8 \text { (final) }\end{array}$ \\
\hline Ejercicio aeróbico & $\begin{array}{l}3 \text { veces por semana, Intensidad entre } 50-70 \% \text { de la frecuencia cardiaca máxima (obtenida } \\
\text { en test de esfuerzo) durante las } 3 \text { primeras semanas y de } 70-80 \% \text { en las últimas } 5 \text { semanas. } \\
\text { Duración de } 60 \text { min aproximadamente }\end{array}$ \\
\hline Ejercicio de sobrecarga & $\begin{array}{l}2 \text { veces por semana, } 12-15 \text { repeticiones a un nivel de esfuerzo de fatiga moderada, 1-3 } \\
\text { series de 8-10 ejercicios de EESS y EEIl }\end{array}$ \\
\hline $\begin{array}{l}\text { Talleres grupales y consejerías } \\
\text { individuales }\end{array}$ & $\begin{array}{l}9 \text { talleres grupales orientados hacia la modificación de estilos de vida/hábitos y control del } \\
\text { estrés, } 1 \text { taller con enfoque de género (exclusivo para mujeres) y } 2 \text { consejerías individuales }\end{array}$ \\
\hline Infraestructura básica & $\begin{array}{l}\text { - } 260 \mathrm{~m}^{2} \text { (tipo de estructura: ladrillo, } 1 \text { piso) } \\
\text { - } 1 \text { gimnasio } \\
\text { - } \quad \text { Sala de reuniones } \\
\text { - } \quad 3 \text { box de relajación } \\
\text { boluación (2 clínico, } 1 \text { psicólogo) }\end{array}$ \\
\hline $\begin{array}{l}\text { Profesionales de la salud } \\
\text { (horas semanales) }\end{array}$ & $\begin{array}{l}\text { Un médico de } 9 \text { años de experiencia ( } 11 \text { h), otros profesionales con al menos } 5 \text { años de } \\
\text { experiencia: } 2 \text { kinesiólogo(a)s (una jornada } 33 \text { h y una jornada de } 22 \text { h), } 2 \text { enfermero(a)s } \\
\text { (una jornada } 33 \text { h y una jornada de } 22 \text { h), } 1 \text { nutricionista }(2 \mathrm{~h}), 1 \text { psicólogo clínico (Jornada } \\
\text { de } 11 \mathrm{~h} \text { ) }\end{array}$ \\
\hline
\end{tabular}


Tabla 2. Costo anual del Programa de Rehabilitación Cardiovascular

\begin{tabular}{|c|c|c|c|}
\hline \multicolumn{4}{|c|}{ Costeo desde la perspectiva del Servicio de Salud } \\
\hline $\begin{array}{l}\text { Recursos relacionados a la provisión } \\
\text { de la atención sanitaria }\end{array}$ & Unidades & $\begin{array}{l}\text { Valor mensual } \\
\quad(P C L)\end{array}$ & $\begin{array}{l}\text { Total anual } \\
\quad(P C L)\end{array}$ \\
\hline Sueldos profesionales de la salud & NA & 3.836 .333 & 46.035 .996 \\
\hline $\begin{array}{l}\text { Sueldos funcionarios administrativos; } \\
\text { auxiliar paramédico ( } 44 \text { h) y secretaria (22) }\end{array}$ & NA & 421.347 & 5.056 .164 \\
\hline Costos fijos (servicios básicos) & NA & 230.463 & 2.765 .553 \\
\hline Material educativo & 112 & 3.000 & 36.000 \\
\hline Gasto en capacitación & 1 & 460.000 & 460.000 \\
\hline $\begin{array}{l}\text { Recursos de mantenimiento anual } \\
\text { según años de vida útil }\end{array}$ & Total & $\begin{array}{l}\text { Vida útil años } \\
\text { (promedio) }\end{array}$ & $\begin{array}{l}\text { Valor anual } \\
\text { (PCL) }\end{array}$ \\
\hline Infraestructura & 37.763 .700 & 50 & 755.274 \\
\hline Mobiliario e instalaciones & 5.631 .625 & 7 & 806.213 \\
\hline Implementos e insumos clínicos & 39.910 .282 & 8 & 8.304 .465 \\
\hline Total gasto anual & & & 64.407 .065 \\
\hline
\end{tabular}

*NA: no aplica; PCL: Pesos chilenos.

Tabla 3. Estimación de los Años de Vida Perdidos por Muerte Prematura para la población del SSMN y los Años de Vida Ganados (AVG) tras un PRC

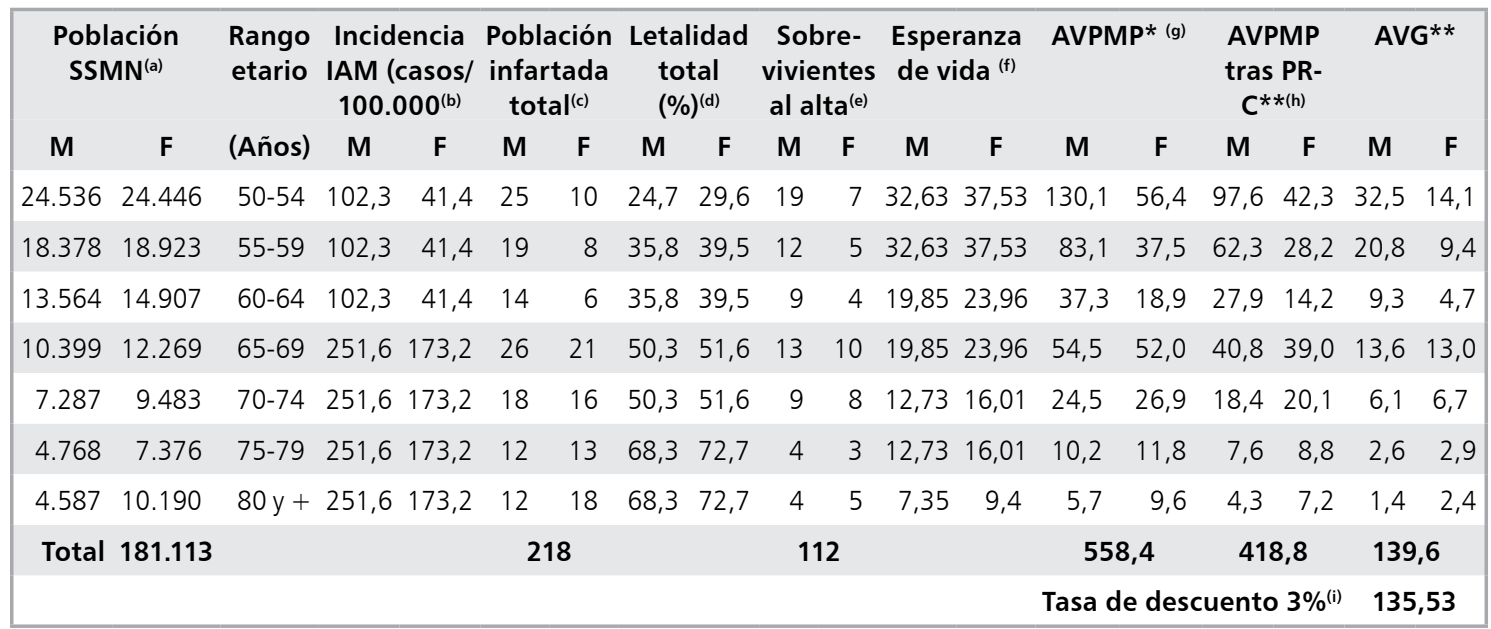

M: Masculino, F: Femenino, AVPMP: Años de vida por muerte prematura. *Calculados para una mortalidad tardía (24 meses) tras trombolisis de $21 \%$. ${ }^{*}$ Calculado en base a (sobrevivientes $\times$ mortalidad/100) $\times 0,75 \times$ esperanza de vida. (a)Proyección de población 2005-2020 (actualizado en agosto de 2010). Proyección población 2012 por grupo de edad, según Región, Servicio de Salud, comuna y sexo. Obtenido de DEIS (sitio web). ${ }^{(b)}$ Reporte de vigilancia de enfermedades no transmisibles (2011). Departamento de Epidemiología. División Planificación Sanitaria. Subsecretaría de Salud Pública. ${ }^{(c)}$ Cálculo en base a: (población por rango etario/100.000) $\times$ incidencia. ${ }^{(d)}$ Incidencia y letalidad por infarto agudo al miocardio en Chile 2001 2007. Carolina Nazzal y Faustino Tomás Alonso. Rev Med Chile 2011; 139: 1253-60. (e)Cálculo en base a: población infartada (población infartada $\times$ letalidad/100). (f)Esperanza de vida por región, grupo etario y sexo. Demografía 1992-2002. Instituto Nacional de Estadística (INE) Publicado 2006. ${ }^{(g)}$ Cálculo con base en: (sobrevivientes $\times$ mortalidad/100) $\times$ esperanza de vida. (h) Considerando una reducción de la mortalidad tardía de $25 \%$. ${ }^{(i)}$ Calculado $A V G /(1+r) \wedge t$; $t=1$ año. 
de cada alternativa. Esto refleja el ICER asociada al PRC presentado previamente teniendo un valor de CLP \$ 475.209,72/AVG. Dentro de un plano cartesiano de costo efectividad (Figura 2) este ICER se posiciona en el cuadrante superior derecho, donde con mayores costos hay mayor efectividad, por lo tanto, la elección de implementar un PRC dependerá de la máxima disposición a pagar (o aceptar) por los efectos en la sobrevida. Al ser este estudio de carácter teórico se medirá su impacto con base a un umbral. La Razón Incremental Costo Efectividad (ICER) de \$475.209,72/AVG es menor a un PIB per cápita 2013 (USD 15732 según datos de Banco Mundial. En CLP \$ 7.792.230,03 [según promedio de dólar de CLP\$495,31 observado por SII en 2013]).

Usando el PIB per cápita como umbral de referencia, esta intervención parece ser muy costo efectiva.

\section{Evaluación de la incertidumbre}

Se consideró diferentes estimaciones y supuestos para obtener la población a tratar (Figura 1). En este reporte, además de las variaciones propias de la incidencia y letalidad en sexo y edad, se realizó un análisis de sensibilidad de las tendencias epidemiológicas. Según un estudio nacional, la incidencia en 6 años, aumentó 9,2\% en el grupo entre 55 y 64 años, mientras que el grupo entre 65 y 74 años disminuyó 11,3\%. Se consideró un cambio anual proporcional. En cuanto a la letalidad, disminuyó $0,7 \%$ anual considerando todos los grupos etarios ${ }^{23}$.

Si consideramos un cambio en la incidencia o en la letalidad, la máxima demanda anual estimada, será 95 y 122 respectivamente. Ambos valores menores de la capacidad máxima estimada para el programa modelo (260 personas). Sin embargo, si consideramos un cambio simultáneo de la incidencia y letalidad, la demanda sobrepasa la capacidad total del centro el sexto año, con 291 personas.

\section{Incertidumbre de parámetros}

El Análisis de Sensibilidad Univariable consideró la variabilidad de los parámetros incidencia, letalidad, derivación y eficacia. El factor más influyente es el cambio en la derivación, estimando una efectividad de 54,21 AVG (40\% de derivación) y de 20,32 AVG (15\% de derivación). Por lo tanto, según el análisis univariable, el ICER en el peor

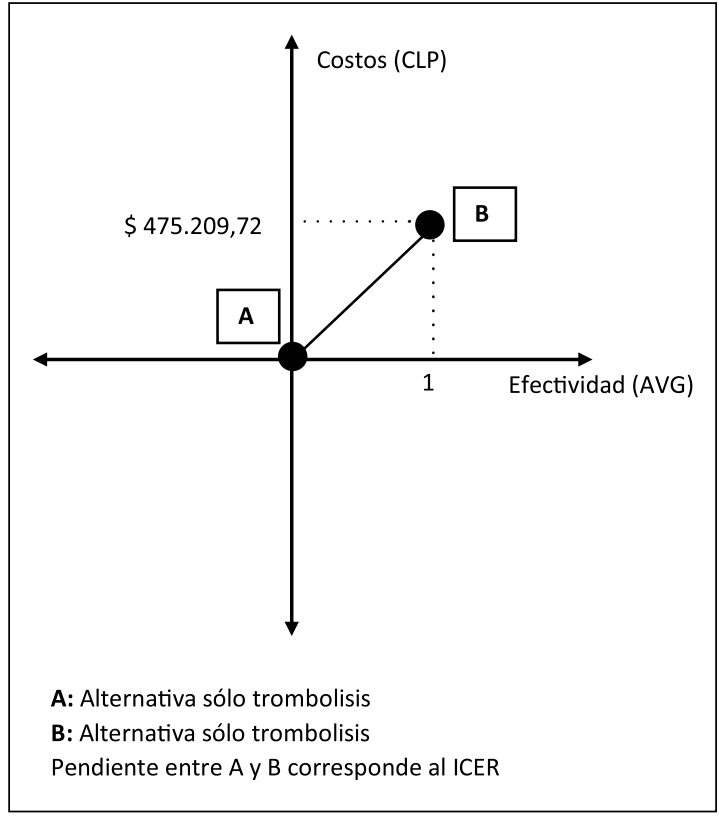

Figura 2. Ubicación ICER en plano cartesiano.

de los casos alcanzaría un valor de \$3.168.141,5/ AVG. Esto corresponde a 40,66\% del PIB per cápita 2013 (Figura 3), lo que continúa siendo muy costo efectivo.

Al analizar la conjugación de parámetros como la derivación y la adherencia al programa obtenemos:

- Derivación $100 \%$ y adherencia 50\%: implica una disminución a 67,5 AVG, con una ICER de $\$ 954.522 / \mathrm{AVG}$, lo que corresponde a $12,25 \%$ del PIB per cápita 2013.

- Derivación $50 \%$ y adherencia 50\%: implica una disminución a 33,7 AVG, con una ICER de $\$ 1.909 .044 / \mathrm{AVG}$, lo que corresponde a $24,4 \%$ del PIB per cápita 2013.

- Derivación $15 \%$ y adherencia $50 \%$ : se obtienen 10,1 AVG, con un ICER de \$6.636.480/AVG, lo que representa $81,66 \%$ del PIB per cápita 2013.

\section{Discusión}

El ICER obtenido indica que un PRC basado en guías clínicas internacionales parece ser muy costo-efectivo en Chile. El costo invertido por año 

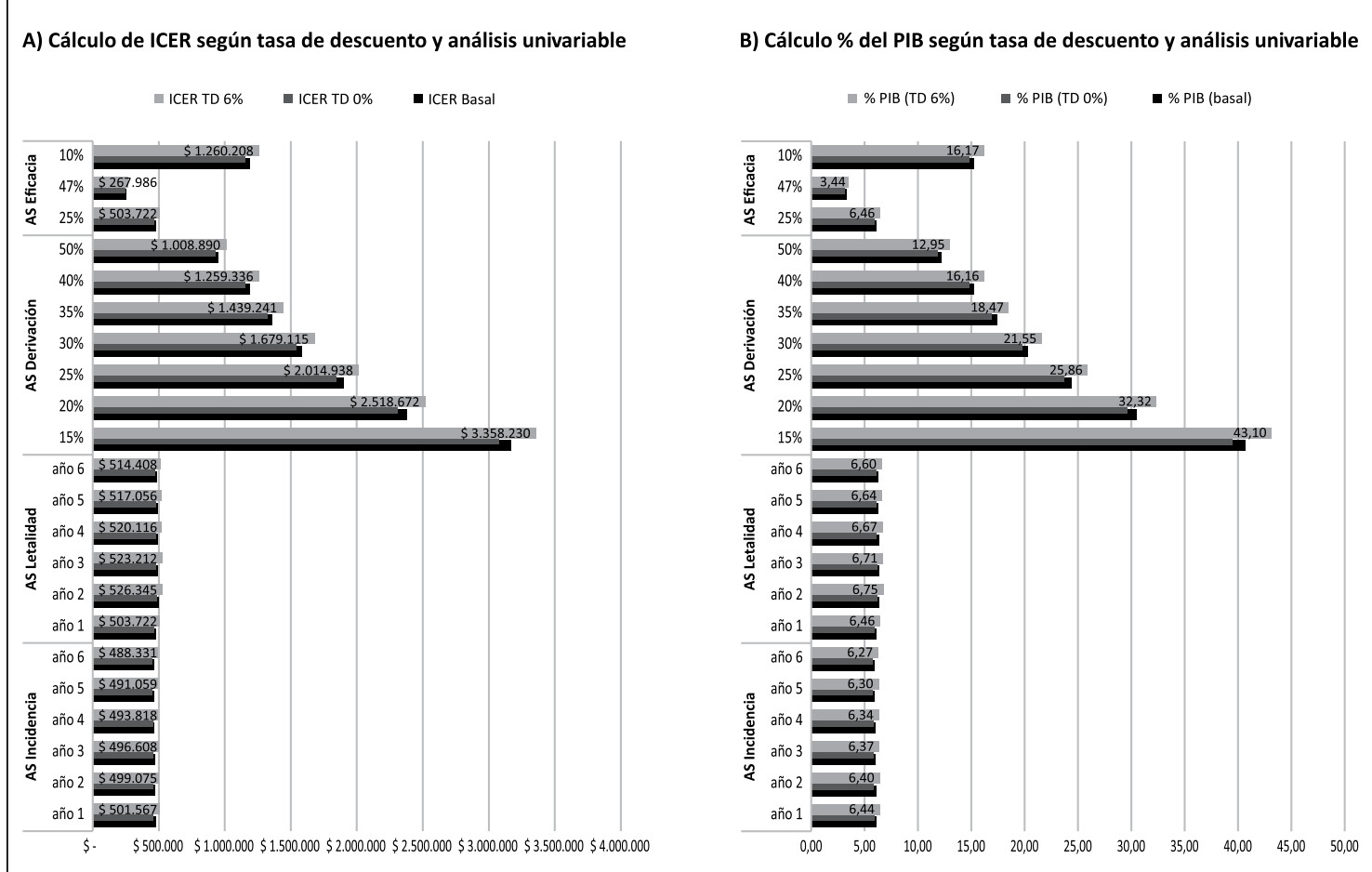

Figura 3. A) Análisis de sensibilidad univariable, efecto en ICER, según tasa de descuento; B) Análisis de sensibilidad univariable, efecto en \% del PIB, según tasa de descuento. AS: Análisis de sensibilidad.

de vida ganado es aproximadamente de $6 \%$ de un PIB per cápita (Banco Mundial, 2013). Incluso considerando la variabilidad de parámetros, la estimación continúa siendo costo efectiva, por ejemplo, Gassner et al. ${ }^{5}$ plantea que en Australia la derivación corresponde varía entre 15-35\%, y estimamos que en Chile la tasa de derivación actual al PRC disponible en el SSMN es de 20\% (datos sin publicar). Sin embargo, de ser sub utilizados los recursos, el programa continúa siendo muy costo efectivo, alcanzando con una derivación de $15 \%$ un ICER que corresponde a 40,7\% del PIB per cápita.

Por otro lado, en la implementación real de un centro de rehabilitación cardiaca su población objetivo puede ampliarse a otros beneficiarios no incluidos en este estudio.

Con respecto a los costos netos (diferencia entre gastos y ahorros/ganancias), se consideró sólo los gastos del PRC, lo que se traduce en una sobre-estimación del costo total, ya que no se calculó el ahorro atribuible a la disminución de la tasa de re-infarto, y los gastos de hospitalización asociados. Por ejemplo, la hospitalización en unidad de tratamiento intensivo asociado a patologías cardiovasculares, representa un gasto de $\$ 423.300$ por día cama, con un promedio de 6 días de internación ${ }^{25,26}$. Este cálculo puede ser objeto de un estudio futuro.

Sólo consideramos el impacto del PRC en la mortalidad para la estimación de los AVG. Sin embargo, existe evidencia suficiente que avala el impacto del PRC sobre la calidad de vida, la capacidad aeróbica máxima, y los factores de riesgo asociados a la enfermedad coronaria ${ }^{27-29}$, que podría asociarse a la reducción de la discapacidad. Esto último podría ser objetivado en estudios de costo utilidad a través de los Años de Vida Ajustados por Calidad (QALY) y/o Años de Vida Ajustados por Discapacidad (DALY).

Los Objetivos Sanitarios de la Década 20122020 contemplan aumentar en $10 \%$ la sobrevida al primer año del IAM. La implementación de un PRC de enfoque integral es clave para contribuir 
al fortalecimiento del nivel secundario de rehabilitación, y en el cumplimiento de esta meta.

Enfatizamos la necesidad de dirigir la atención de los investigadores hacia sub-grupos tales como mujeres, personas mayores de 65 años, y pacientes con riesgo $\mathrm{CV}$ alto 5 . Existe evidencia que indica que grupos de mayor perfil de riesgo pueden obtener mejores resultados a través de PRC, por lo que son recomendables para esta sub-población ${ }^{30}$. Personas con hipertensión arterial, o diabetes tipo II, pueden beneficiarse en forma significativa ${ }^{31}$.

El ejercicio físico debe ser considerado una herramienta terapéutica en el tratamiento de la enfermedad coronaria. Además un campo laboral consolidado para los kinesiólogos(as). Desde 2006 a 2011, el colegio de kinesiólogos registra 13 kinesiólogos con especialidad en Cardiología y Cirugía Cardiovascular (DENAKE, 2011), es esperable que esta cifra aumente los próximos años.

Agradecimientos: A los kinesiólogos que participaron en la revisión y corrección del programa modelo; Claudia Román, Klga. Especialista Cardiovascular acreditada DENAKE, Complejo Hospitalario San José; Manuel Alvear, Klgo. Especialista Fisiología Aeroespacial, Universidad del Desarrollo y Karen Rouliez, Klga. Programa de Rehabilitación Cardiaca HCUCH, y docente del Diplomado en Kinesiología Cardiorespiratoria. Y a los consultores del área economía; Sylvia Galleguillos, Directora IAS Facultad de Economía y Negocios, Universidad de Chile, y Marianela Castillo, Economista MINSAL.

\section{Referencias}

1. DEIS. Serie de Mortalidad por causas 2000-2010. Actualizado el 2012.

2. Ministerio de Salud, Secretaría de Salud Pública. Reporte de Vigilancia de Enfermedades No Transmisibles (ENT). Recuperado septiembre 2012. Disponible en: http://epi.minsal.cl/epi/0notransmisibles/vent/VENT_ Mercosur_FINAL_17092011.pdf

3. Balady G, Williams M, Ades P, Bittner V, Comoss P, Foody J, et al. Core components of cardiac rehabilitation/secondary prevention programs: 2007 update: a scientific statement from the American Heart Association Exercise, Cardiac Rehabilitation, and Prevention Committee, the Council on Clinical Cardiology; the Councils o. Circulation 2007; 115 (20): 2675-82.
4. Romero T. La rehabilitación cardiaca como punto de partida en la prevención secundaria de la enfermedad coronaria. Rev Med Chile [revista en la Internet]. 2000 agosto [citado el 31 de marzo de 2013]; 128 (8): 923-34. Disponible en: http://www.scielo.cl/scielo.php?script=sci_arttext\&pid=S0034-98872000000800013\&lng=es.

5. Gassner L, Dunn S, Piller N. Aerobic exercise and the post myocardial infarction patient: a review of the literature. Heart \& Lung: The Journal of Acute and Critical Care 2003; 32 (4): 258-65.

6. Taylor R, Brown A, Ebrahim S, Jolliffe J, Noorani H, Rees K, et al. Exercise-based rehabilitation for patients with coronary heart disease: systematic review and meta-analysis of randomized controlled trials. The American journal of medicine 2004; 116 (10): 682-92.

7. O’ Connor G, Buring J, Yusuf S. An Overview of Randomized Trials of Rehabilitation With Exercise After Myocardial Infarction. Circulation1989; 80: 234-44.

8. Jolliffe J, Rees K, Taylor R, Thompson D, Oldridge N, Ebrahim S. Exercise-based rehabilitation for coronary heart disease. Cochrane Database of Systematic Reviews. The Cochrane Library, 2011; 03.

9. Clack A, Hartling L, Vandermeer B, McAlister F. Meta-Analysis: Secondary Prevention Programs for Patients with Coronary Artery disease. American College of Physicians 2005; 659-74.

10. Lawler P, Filion K, Eisenberg M. Efficacy of exercise-based cardiac rehabilitation post-myocardial infarction: A systematic review and meta-analysis of randomized controlled trials. Am Heart J 2011; (162): 571-84.

11. Maroto M, Artigao R, Morales D, Zarzosa C, Abraira V. Rehabilitación cardiaca en pacientes con infarto de miocardio. Resultado tras 10 años de seguimiento. Revista española de cardiología 2005; 58 (10): 1181-7.

12. Karmishold K, Gotzsche P. Physical activity for secondary prevention of disease. Danish Medical Bulletin 2005; 52 (2): 90-4.

13. Yu Ch-M, Lau Ch-P, Chau J, McGhee S, Kong S-L, Man-Yung Cheung B, et al. A Short Course of Cardiac Rehabilitation Program is Higly Cost Effective in Improving Long-Term Quality of Life in Patients With Recent Myocardial Infarction or Percutaneous Coronary Intervention. Archives of Physical Medicine and Rehabilitation 2004; 85: 1915-22.

14. Haskell W, Lee I, Pate R, Powell K, Blair S, Franklin B, et al. Physical Activity and Public Health: Updated Recommendation for Adults from the American College of Sports Medicine and the American Heart Association. Medicine \& science in sport \& exercise 2007; 39 (8): 1423-34.

15. Fletcher G, Balady G, Ezra A, Chaitman B. Excercise 
Standards for testing and training: A Statement for Healthcare Professionals from the American Heart Association. Circulation 2011; 104: 1694-740.

16. Hunt SH, Baker D, Chin M, Cinquegrani M, Feldman A. ACC/AHA Guidelines for evaluation and management of chronic heart failure in adult. A report of the American College of Cardiology/American Heart Asociation Task Force on Practice Guidelines. Circulation 2001; 104: 2996-3007.

17. Taylor R, Watt A, Dalal H, Evans P, Campbell J, Read $\mathrm{K}$, et al. Home-based cardiac rehabilitation versus hospital-based rehabilitation: A cost effectiveness analysis. International Journal of Cardiology 2007; 119: 196-201.

18. Santibáñez C, Pérez-Térzic C, López-Jiménez F, Cortés-Bergoderi M, Araya MV, Burdiat G. Situación actual de la rehabilitación cardiaca en Chile, Rev Med Chile 2012; 140: 561-8.

19. Korenfeld Y, Mendoza-Bastidas C, Saavedra L, Montero-Gómez A, Pérez-Terzic C, Thomas RJ, et al. Current status of cardiac rehabilitation in Latin America and the Caribbean. Am Heart J 2009; 158 (3): 480-7.

20. Briffa T, Eckerman S, Griffiths A, Harris P, Health M, Freeman $\mathrm{S}$, et al. Cost-effectiveness of rehabilitation after an acute coronary event: a randomized controled trial. Medical Journal of Australia 2005; 183 (9): 450-5.

21. Ministerio de Salud. Guía metodológica para la Evaluación Económica de Intervenciones en Salud en Chile, 2013.

22. Ministerio de Salud. Informe Final: Estudio Costo efectividad de Intervenciones en Salud, 2010. Disponible en: http://desal.minsal.cl/DOCUMENTOS/PDF/GES/1.2/ 01CostoEfectividad.pdf

23. Nassal C, Alonso F. Incidencia y letalidad por Infarto Agudo al Miocardio en Chile: 2001-2007. Rev Med Chile 2011; 139: 1253-60.

24. Martínez A, Nassal C, Fajuri N, Barra L, Mayerson A,
Cavada G, et al. Mortalidad post infarto del miocardio en Chile: Comparación de los registros de angioplastía primaria versus trombolisis. Rev Chil Cardiol 2010; 29: 29-36.

25. Alvear S, Canteros J, Rodríguez P. Estudio retrospectivo de costos de tratamientos intensivos por paciente y día cama. Rev Med Chile 2010; 138: 558-66.

26. Alvear S, Canteros J, Jara J, Rodríguez P. Costos reales de tratamientos intensivos por paciente y día cama. Rev Med Chile 2013; 141: 202-8.

27. Colin W, Alison E. Cardiac rehabilitation and quality of life: A systematic review. International Journal of Nursing Studies 2012; (49): 755-71.

28. Griffo R, Ambrossetti M, Tramarin R, Fattirolli F, Temporelli $\mathrm{P}$, Vestri A, et al. Effective secondary prevention through cardiac rehabilitation after coronary revascularization and predictors of poor adherence to lifestyle modification and medication. Results of the ICAROS Survey. International Journal of Cardiology (2012/in press).

29. Olsen A, Boas A, Rasmussen S, Frederiksen M, Abadini $S$, Appel J, et al. Hospital-based comprehensive cardiac rehabilitation versus usual care among patients with congestive heart failure, ischemic heart disease, or high risk of ischemic heart disease: 12-Month results of a randomized clinical trial. Am Heart J 2008; (155): 1106-13.

30. Schwaab B, Waldmann A, Katalinic A, Sheikhzadeh A, Raspe H. In-patient cardiac rehabilitation versus medical care-a prospective multicenter controlled 12 months follow-up in patients with coronary heart disease. Eur J Cardiovasc Prev Rehabil 2011; 18 (4): 581-6.

31. O'Connor C, Whellan D, Lee K, Keteyian S, Cooper L, Ellis S, et al. Efficacy and Safety of Exercise Training in Patients With Chronic Heart Failure HF-ACTION Randomized Controlled Trial. JAMA 2009; 301 (14): 1439-50. 